\title{
ARRECIFES ARTIFICIALES: ESTRUCTURAS LLENAS DE VIDA
}

\section{(ARTIFICIAL REEFS: A LIVE STRUCTURES)}

\author{
José Luis Gayo Romero, Ing. Técnico de Obras Públicas
}

Dirección General de Costas. Ministerio de Medio Ambiente

\section{ESPAÑA}

Fecha de recepción: $30-\mathrm{X}-98$

\section{SUMMARY}

The increase of infraction on the national fisherie arts regulations and overfishing on natural fisherie are some of the main problems of the spanish shoreline.

Civil engeniering with other disciplines can support some solutions trough the creation of artificial reefs. A review of the methodology for the construction of artificial reefs is presented in this paper.

\section{Introducción y antecedentes}

Un arrecife artificial es un conjunto de estructuras que, fondeadas en el medio marino, introducen en éste una diversificación de ecosistemas, creando áreas o zonas de repoblación marina natural.

Los objetivos principales de este tipo de obras básicamente son dos: la reproducción biológica y la protección de los fondos marinos frente a artes de pesca indiscriminada, como el arrastre.

Los resultados obtenidos se centran en el aumento de la productividad biológica, diversidad del ecosistema y creación de zonas de pesca artesanal selectiva, entre otros.
La introducción de elementos atípicos en el medio marino, provoca, con el paso del tiempo, la proliferación y crecimiento de organismos vivos, desarrollo de algas y fauna marina en su entorno; se producen, así, variaciones en el medio, creándose un nuevo conjunto de factores físicos que conforman el medio ambiente, desarrollándose en éste una nueva comunidad biológica biocenosis o potenciando la comunidad original, concentrándola en torno al elemento extraño introducido.

Si en un fondo arenoso introducimos un elemento duro se induce una variación ecológica. Especies hasta entonces no presentes en el sistema aparecen colonizando el sustrato duro, refugiándose y reproduciéndose en él. A su vez, las especies superiores de la cadena trófica son atraídas a la 
zona favoreciendo el reciclaje de energía e incrementando el valor ecológico y, en su caso, comercial de la nueva comunidad.

Los primeros estudios y experiencias relativos a este tipo de estructuras se desarrollaron en países como Japón y Estados Unidos, siendo ambos actualmente los más avanzados en su diseño y construcción. En el Mediterráneo destacan las experiencias en este campo de los técnicos españoles.

Los primeros arrecifes artificiales fueron realizados mediante el hundimiento o fondeo de elementos constituidos por diversos materiales; las experiencias japonesas utilizaban elementos formados por estructuras de madera, elementos plásticos, hormigones, aceros y, posteriormente, fibras de vidrio, etc., dirigidas, específicamente, al aumento de la producción pesquera y a la protección de alevines.

Las experiencias americanas, sin embargo, han utilizado generalmente materiales de desecho como chatarras de carrocerías de coches, barcos y aviones de desguace, neumáticos, etc., dirigidos, especialmente, al aumento de la pesca deportiva.
En Europa se ha adoptado el sistema japonés, que permite la protección de los fondos de la pesca de arrastre, potenciando la pesca artesanal y minimizando la mortandad por exceso de la misma, especialmente en la época donde abundan ejemplares jóvenes.

\section{Concepción global de un proyecto de arrecife arti- ficial}

La creación de un nuevo arrecife es un proceso complejo que consta de diversas fases y que están concatenadas espacial y temporalmente (Figura 1).

\section{Estudios previos}

La elección de las zonas apropiadas para fondear las estructuras debe de realizarse tras analizar una serie de factores de tipo batimétrico, físicos, morfológicos, geológicos, ecológicos, pesquero-comerciales, constructivos, etc.

El primer paso a la hora de proyectar un arrecife artificial es definir las zonas que se pretenden proteger, se deberán estudiar los diferentes caladeros que existen, así como los

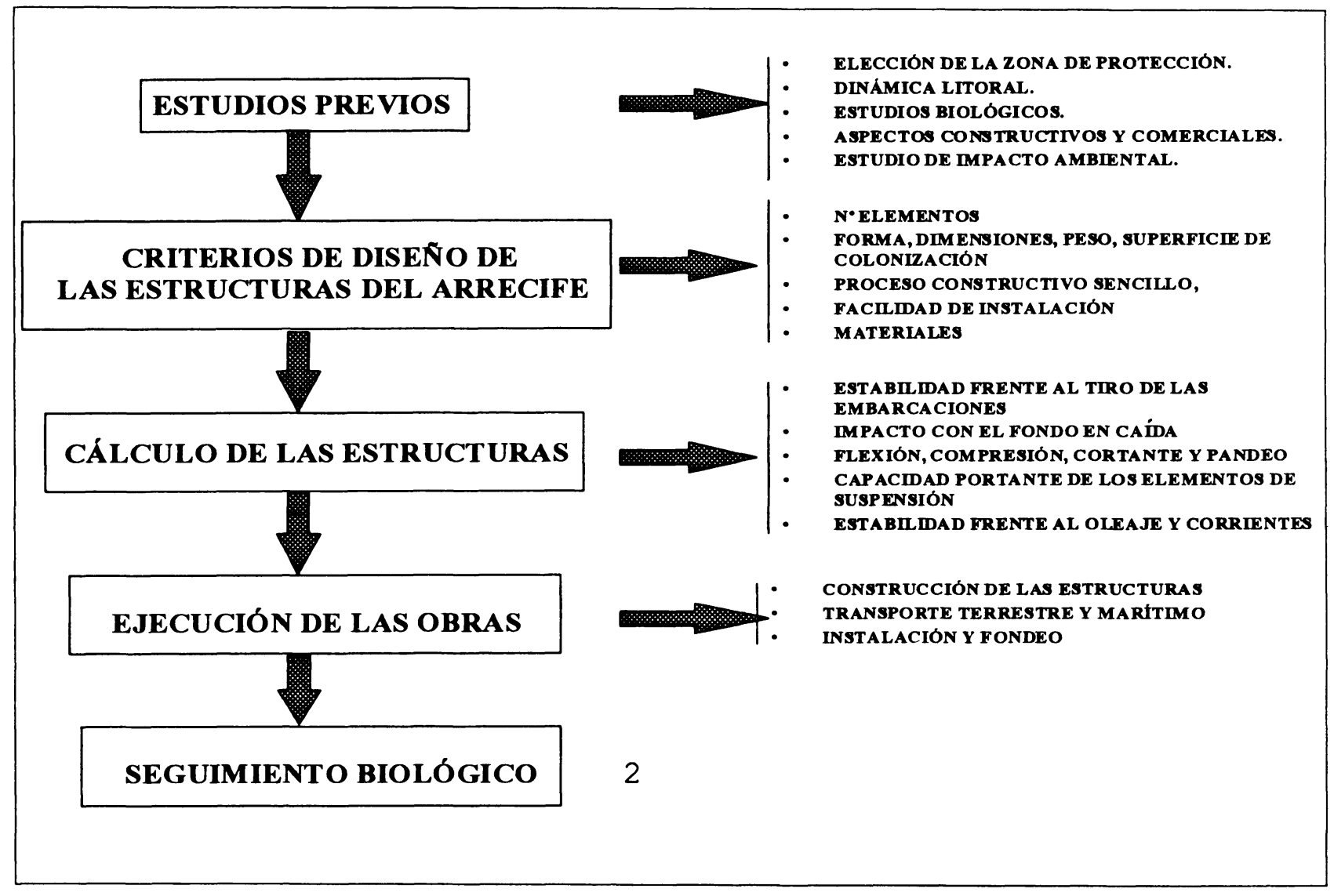

Figura 1.- Esquema global de un proyecto de arrecifes artificiales. 
lances de arrastre ejecutados por los barcos que faenan ilegalmente dentro de profundidades menores a $50 \mathrm{~m}$, para detectar sus trayectorias.

Debido al carácter ilegal o furtivo, no siempre resulta fácil definir las trayectorias seguidas por los barcos, por ello, en estos casos, se deberán investigar por otros métodos:

Se pueden detectar las trayectorias mediante buceadores o mediante Side Scam Sonar y, así, estudiar la situación más adecuada de los módulos para realizar su función de obstáculo.

También se detectan las trayectorias preguntando en las cofradías de pescadores, ya que el sector artesanal (cerco, palangre, trasmallo, cadups, curricán, etc.) es el más afectado por el uso de artes no selectivas, como el arrastre y, a su vez, el más interesado en proteger su pesca.

Con estas medidas se trata también de reducir el conflicto entre los distintos sectores pesqueros, realizando una ordenación pesquera.

La experiencia demuestra que los arrastreros ilegales, finalmente, descubren la situación de los arrecifes, provocando, en muchos casos, la desestabilización, e incluso el arrastre de los módulos intencionadamente, con el fin de abrir huecos en las barreras de protección o, simplemente, levantan las puertas del arte que arrastran por el fondo para sortearlas, por ello es conveniente estudiar la distancia mínima rentable del lance para cada zona, de manera que aunque sortearan una barrera, la longitud recorrida hasta la siguiente fuese tal que la pesca resultara mínima, no siendo rentable, comercialmente, realizar esos lances.

\subsection{Profundidad de la instalación}

Desde el punto de vista de la ingeniería de costas, la profundidad a la que se sitúe la instalación no deberá interrumpir o alterar los procesos de Dinámica Litoral y, en especial, el transporte de sedimentos. La profundidad idónea será función directa de la iluminación de la zona sumergida, penetración de la luz, turbidez de las aguas, etc., de manera que permitan el desarrollo de organismos sésiles y algas fotófilas en las estructuras de reproducción.

Se puede considerar como profundidades óptimas aquéllas que abarcan hasta la zona final del piso infralitoral entre las batimétricas $-15 \mathrm{~m}$ y $-35 \mathrm{~m}$ en general, debiéndose estudiar las características particulares en cada caso concreto.

\subsection{Batimetrias y pendientes de los fondos}

Es fundamental, dentro de los estudios y toma de datos previos, la realización de una batimetría de la zona de estudio. Ésta, proporcionará adecuada información sobre el relieve y las pendientes de los fondos marinos donde se implantará la instalación del conjunto de elementos.

La pendiente deberá ser mínima para proporcionar la suficiente estabilidad a las estructuras. El relieve de los fondos nos ayuda a prever los posibles pasos o entradas por los que podrían acceder las redes al arrecife, estos pasos serán cubiertos con estructuras de protección que dificulten el acceso.

\subsection{Consideraciones geológicas}

El conocimiento del subsuelo marino y su morfología es fundamental desde el punto de vista del diseño de las estructuras.

Es conveniente conocer tanto la capacidad portante del fondo marino como los materiales que lo forman, así como las isopacas de los sedimentos existentes, ya que la estructura podría quedar embebida dentro del sedimento (caso de fondos fangosos) o mal colocada, no cumpliendo la función para la cual fue diseñada.

\subsection{Aspectos ecológicos previos}

La instalación del arrecife pretende crear un nuevo biótopo introduciendo un sustrato duro que dará lugar a la colonización progresiva de comunidades biológicas diferentes de las que existían inicialmente. Por ello se debe tener en cuenta que la zona de instalación deberá estar lo más alejada posible de zonas rocosas, los mejores fondos serán los de sedimentos no consolidados. Es fundamental que en la zona de fondeo existan aportes de nutrientes abundantes y no se localicen emisarios submarinos en las proximidades.

Muy importante es el estudio del posible efecto de concentración, ya que el arrecife puede actuar como un elemento de concentración de las especies de la zona en puntos concretos, con la consiguiente disminución de la pesca en los caladeros tradicionales cercanos.

Se deberán realizar levantamientos de cartografía biológica, distribución de comunidades y tipos de fondos, encaminados a la descripción del estado actual del sistema en fondos, (comunidades naturales y recursos pesqueros explotables), identificando las diferentes comunidades y valorando ecológicamente las mismas, así como la capacidad de explotación de los bancos naturales existentes en las zonas del Estudio.

\subsection{Aspectos constructivos y comerciales}

Constructivamente conviene situar el arrecife artificial a distancias relativamente cercanas a puertos con suficiente capacidad para almacenar o acopiar las estructuras; co- 
mercialmente también hay que considerar la proximidad del arrecife a puertos pesqueros.

\section{Características y criterios de diseño de las estructuras del arrecife}

Cuando el técnico se plantea el diseño de las estructuras arrecifales debe de tener en cuenta ciertas consideraciones.

Podemos diferenciar tres tipos básicos de estructuras, dependiendo de su función (Fotografias 1-4) :

\section{. Estructuras de Protección}

. Estructuras de Reproducción

. Estructuras Mixtas
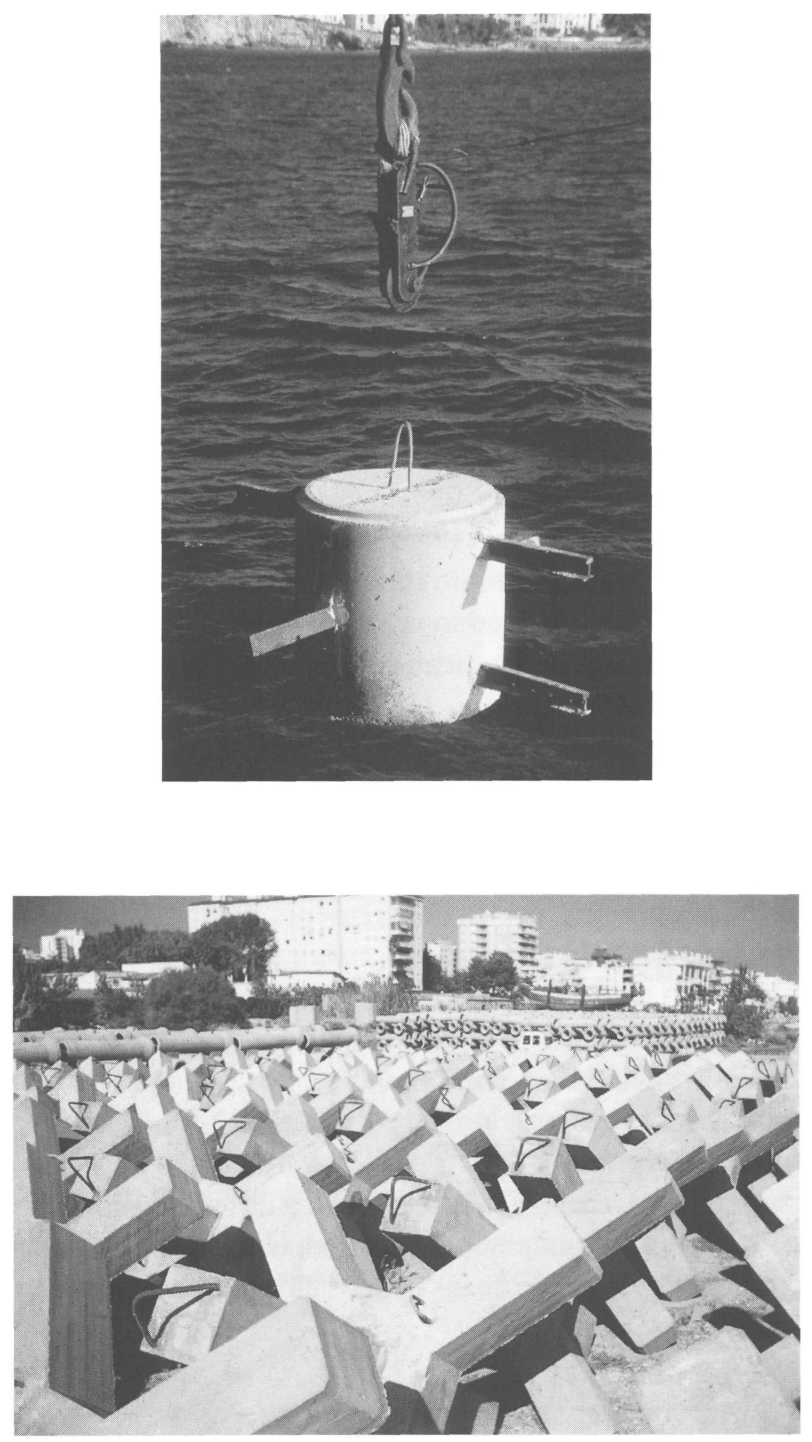

Estructuras de Protección.
Las estructuras de Protección son aquéllas que, por su forma, peso y estabilidad están indicadas para disuadir o impedir el acceso de barcos con artes como el arrastre, a la zona de reproducción.

El peso de la estructura de protección se calculará en función de la potencia que puedan desarrollar los barcos de la flota de arrastre susceptible de faenar en la zona de protección, de manera que presente la suficiente estabilidad al empuje, deslizamiento y vuelco. Su diseño y forma presentará los suficientes puntos de enganche y robustez que permitan el bloqueo, estorbo o desgarramiento de las redes de arrastre, impidiendo el avance. El peso óptimo de este tipo de elementos se estima entre 3 y $7 \mathrm{t}$, en seco.

Las estructuras de Reproducción son aquéllas que tienen por finalidad el desarrollo y regeneración de la fauna

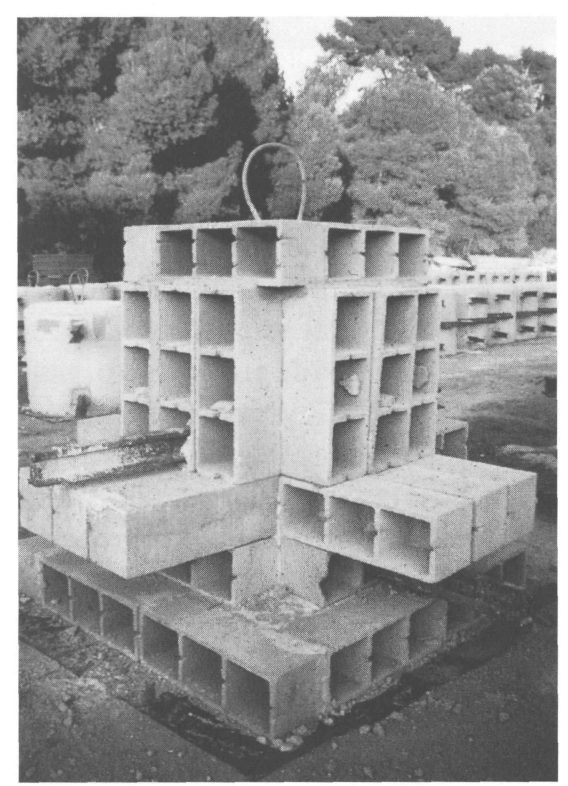

Estructura Mixta.

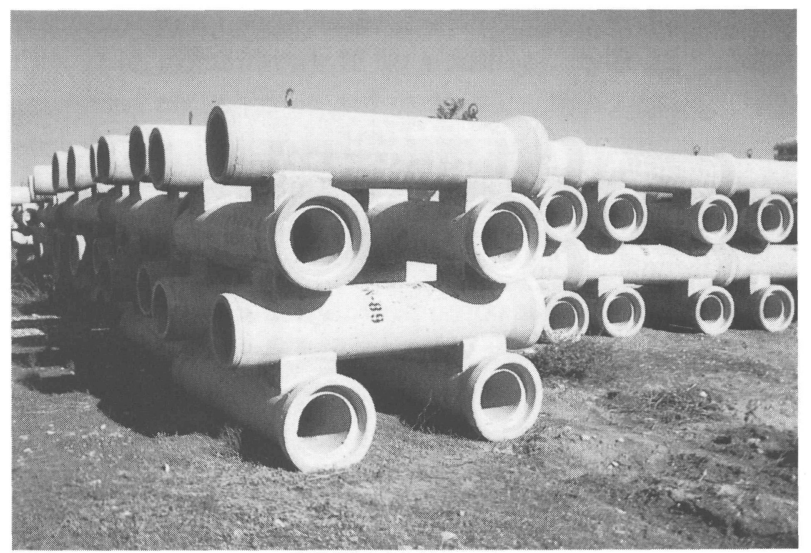

Estructura de Reproducción. 


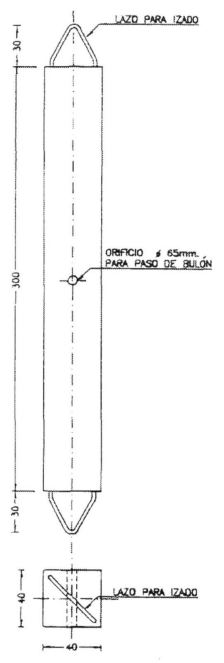

ELEMENTO CU. A.1

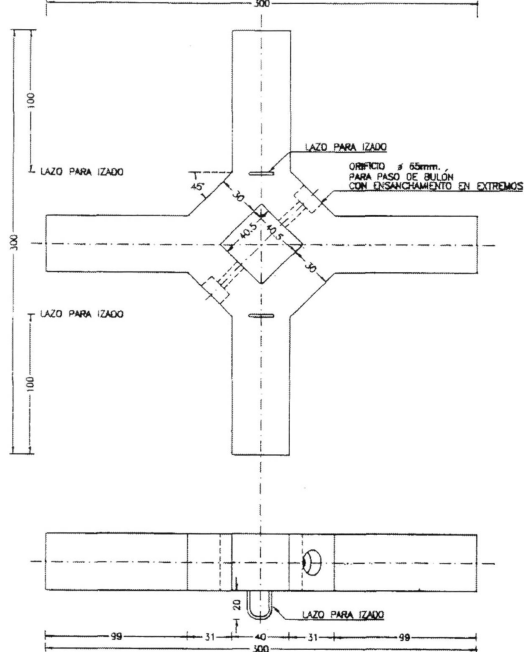

ELEMENTO CU. A.2
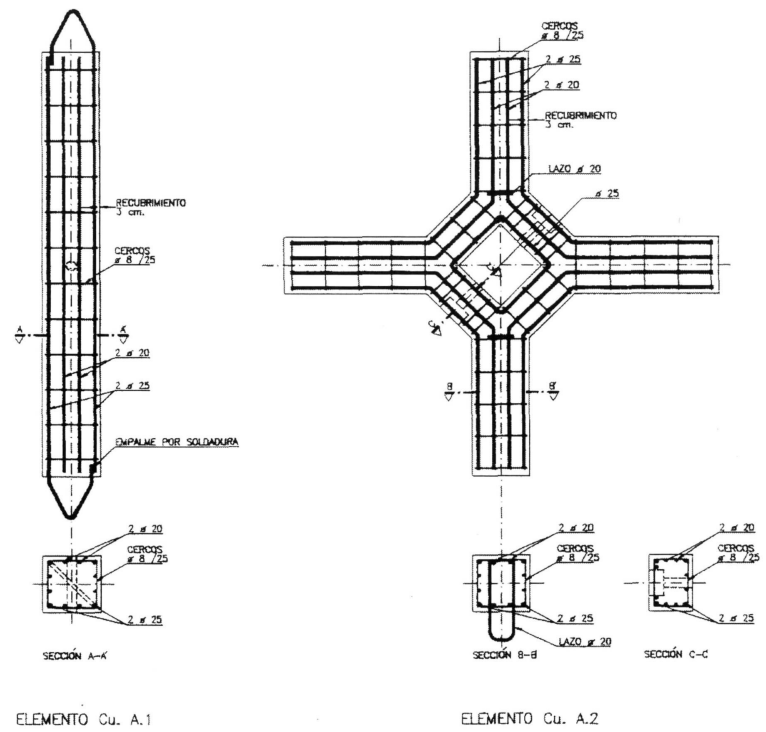

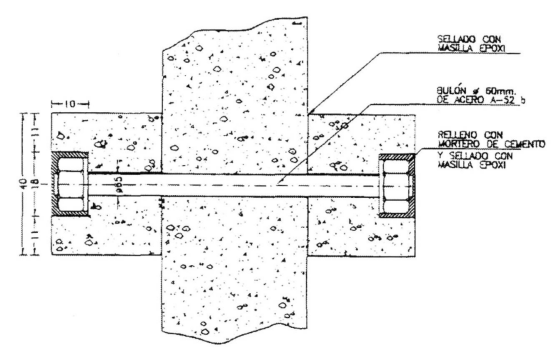

OETALLE DE SUJECIÓN MEDIANTE BULÓN
ELEMENTO CU. A.

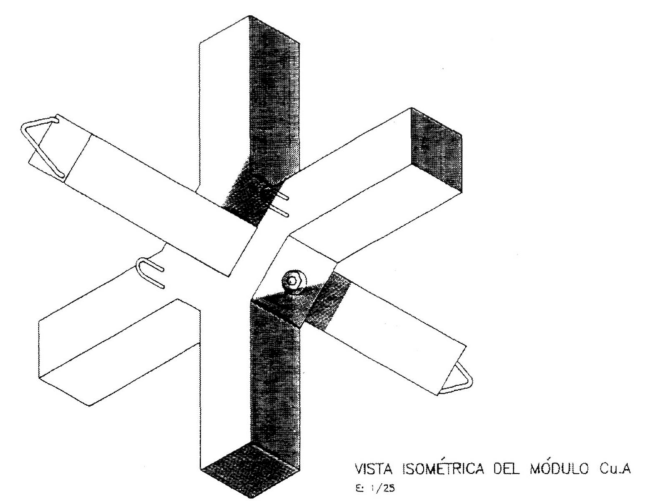

Estructura de Protección. Elementos.

marina, favoreciendo la repoblación, puesta y reproducción de las diferentes especies que lo colonicen.

Las características principales de estos elementos son: peso suficiente para permitir la estabilidad, además de formas que favorezcan el antiarrastre. Los elementos presentarán el mayor número de superficies, huecos y planos horizontales, verticales e inclinados, que permitan la entrada de la luz, corrientes de agua y oxigenación suficientes para favorecer la productividad y colonización de las especies.

Existe un tercer tipo denominado Mixto que conjunta las cualidades de los dos anteriores, actuando como protector-reproductor .

Como características intrínsecas a los dos tipos de estructuras se pueden citar:

(c) Consejo Superior de Investigaciones Científicas Licencia Creative Commons 3.0 España (by-nc)
. El número de elementos que formarán el arrecife vendrá en función delárea de la zona a proteger y de los recorridos de los lances de los arrastreros.

. La vida útil de la estructura se estima entre 30 y 50 años.

. Las estructuras serán eficientes, funcionales y económicas.

. Los materiales que se emplearán en su construcción deben ser limpios (esto es, no contaminantes), tendrán suficiente durabilidad, su puesta en obra y ejecución se realizará con facilidady, fundamentalmente, serán económicos y poco corrosivos. Hoy en día el principal material de construcción es el hormigón, ya que cumple con las principales exigencias requeridas.

. Los módulos deberán tener formas y dimensiones fá- 


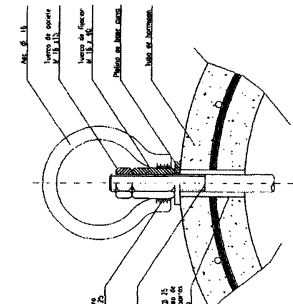

目目望新
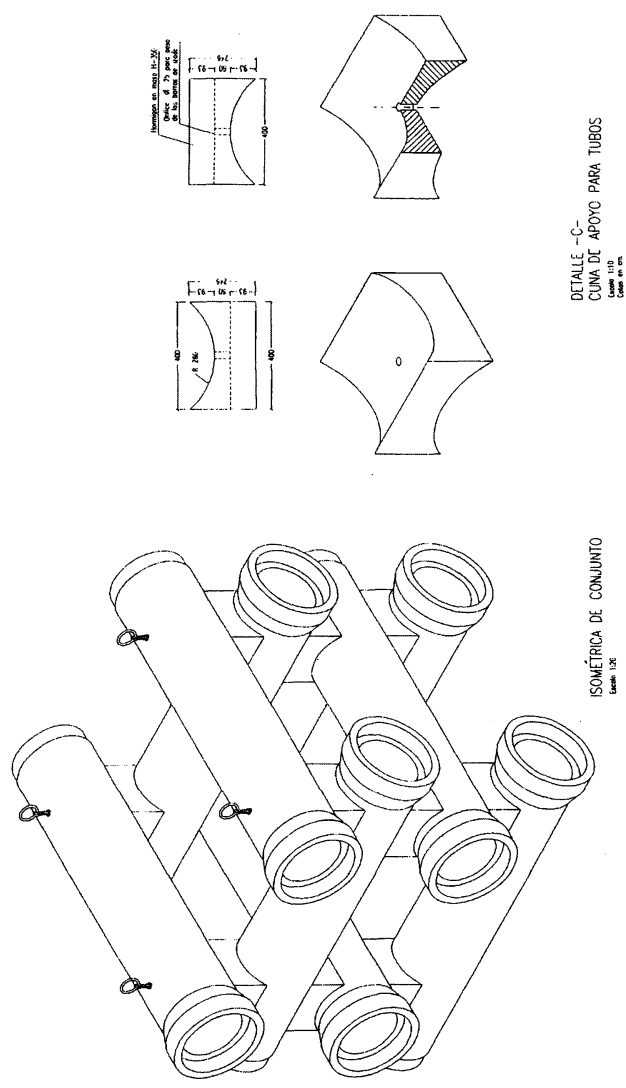

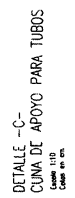

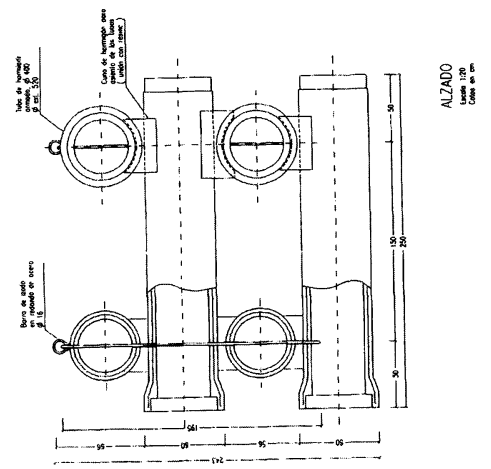

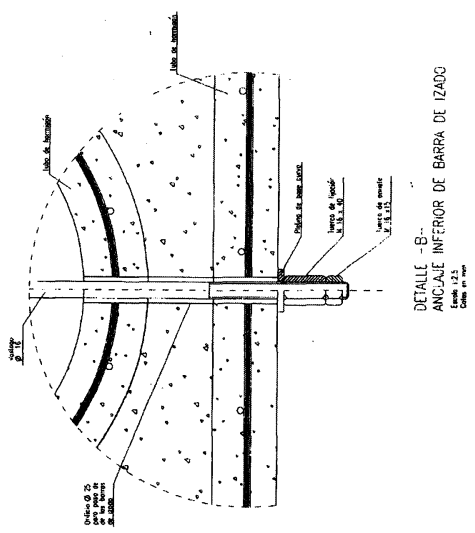

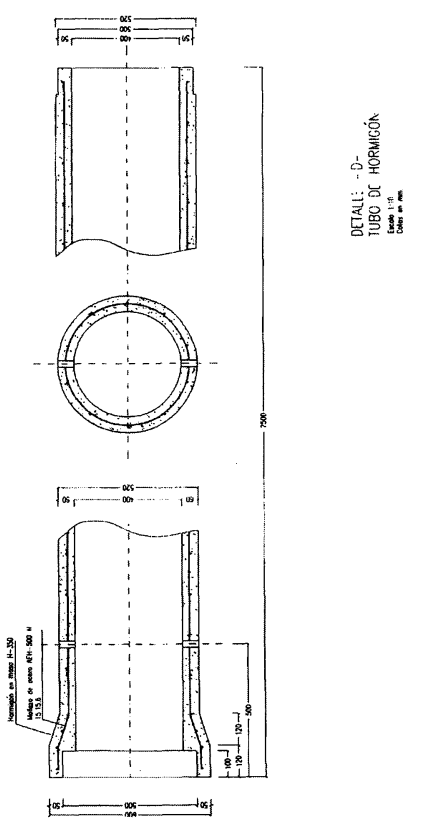

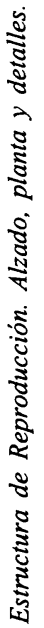


cilmente ejecutables y sencillas, permitiendo su construcción prefabricada y ensamblaje entre sí.

. Las superficies de apoyo de la estructura se estudiarán de manera que eviten el hundimiento de ésta en los sedimentos del subsuelo marino.

. Se deberá estudiar la posible alteración de la dinámica litoral y la afección de la obra a la navegación comercial.

\subsection{Materiales empleados en la construcción}

Entre los posibles materiales a utilizar en la construcción de los elementos arrecifales destaca el hormigón por las ventajas que proporciona frente a otros posibles, pero debe cumplir los siguientes condicionantes:

Los conglomerantes hidráulicos utilizados para la puesta en obra de las estructuras deberán cumplir la Normativa vigente, Pliego de Prescripciones Técnicas Generales para la recepción de Conglomerantes Hidráulicos (RC 88) y la Instrucción para el proyecto y ejecución de obras de hormigón en masa o armado $\mathrm{EH}-91$.
Los cementos a utilizar s erán, principalmente, puzolánicos y resistentes al agua de mar, para que el hormigón producido resista la agresión de las sales que el agua marina lleva disueltas; su denominación cumplirá con la norma UNE 80 - 301.

Es muy importante que el cemento presente un bajo contenido en aluminato tricálcico, ya que éste reacciona con los sulfatos para formar sulfoaluminato cálcico hidratado, cuyas moléculas aumentan de volumen, formando un compuesto, de carácter expansivo, que provoca la disgregación del hormigón. El contenido en aluminato tricálcico será inferior al $8 \%$ y tendrán la denominación MR.

Los cementos resistentes a sulfatos y agua de mar se elegirán por su composición y por la constitución de su clínker; en cualquier caso cumplirán las especificaciones recogidas en la tabla 1 de la norma UNE 80-303-86.

La adición de puzolanas, tanto naturales (lava volcánica) como artificiales (escoria de alto horno), proporciona propiedades resistentes al ataque de los sulfatos. En este

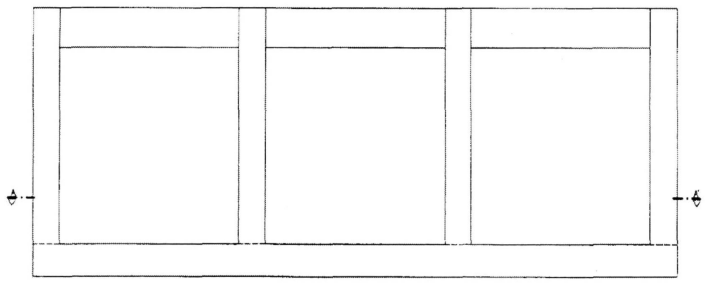

ALZADO LATERAL

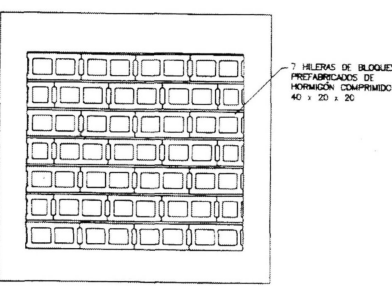

ALZADO FRONTAL

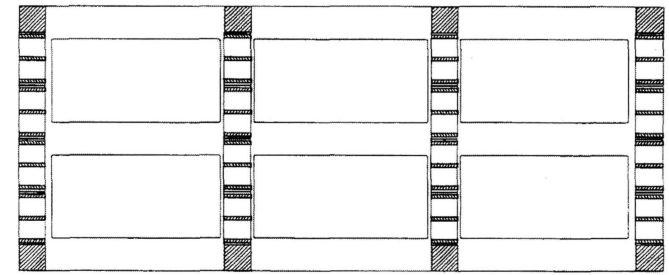

SECCIÓN A-A'

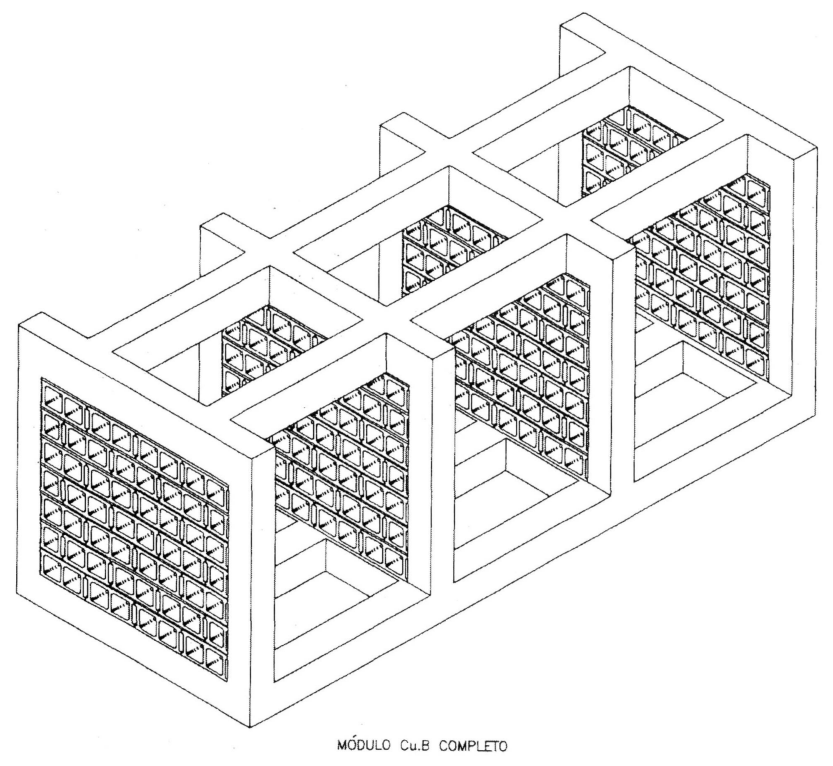


sentido, es muy recomendable la utilización de cementos puzolánicos de tipo IV, según la denominación UNE 80-301.

Se recomienda una dosificación no inferior a $350 \mathrm{~kg}$ de cemento por metro cúbico de consistencia plástica.

La resistencia característica del hormigón no será inferior a $200 \mathrm{~kg} / \mathrm{cm}^{2}$ y el tamaño del árido será tal que permita mejorar la compactación: se recomienda un tamaño máximo de $20 \mathrm{~mm}$.

Para el amasado del hormigón se recomiendan las indicaciones de la EH-91 y, en cualquier caso, el agua no producirá eflorescencia, agrietamiento o perturbación del fraguado.

Los aditivos, a priori, deben de prohibirse, por la dificultad de controlar su comportamiento en el tiempo una vez sometido a la agresividad marina, salvo que se comprobase, mediante ensayos, la no perturbación de sus características. En caso de armar las estructuras se recomiendan barras corrugadas de acero de $4.200 \mathrm{kp} / \mathrm{cm}^{2}$, como mínimo.

\section{Cálculo de las estructuras}

Antes de iniciar la construcción de las estructuras que compondrán el arrecife artificial es necesario realizar, al menos, los siguientes cálculos para su dimensionamiento.

5.1. Cálculo de la estabilidad de la estructura ante los efectos del oleaje

Para calcular la estabilidad ante el oleaje se parte de los siguientes datos:

- Profundidad mínima a la que se encuentra la estructura (d)

- Altura de ola de cálculo (H)

con la altura de ola de cálculo, se obtiene el período (T) directamente mediante gráficos.

Para realizar los cálculos es conveniente definir la profundidad relativa en la que nos encontramos; ésta viene definida por el cociente entre la profundidad a la que se encuentra la estructura y la longitud de onda.

profundidad de la estructura (d)/ longitud de onda (L):

\begin{tabular}{|c|c|c||}
\hline Profundidad indefinida & Zona de transición & Profundidad reducida \\
\hline $\mathrm{d} / \mathrm{L}<1 / 2$ & $1 / 25<\mathrm{d} / \mathrm{L}<1 / 2$ & $\mathrm{~d} / \mathrm{L}<1 / 25$ \\
$\mathrm{~d} / \mathrm{g} \times \mathrm{T} 2>0,08$ & $0,08>\mathrm{d} / \mathrm{g} \times \mathrm{T} 2>0,0025$ & $\mathrm{~d} / \mathrm{g} \times \mathrm{T} 2<0,0025$ \\
$\mathrm{v} \cong 0$ & $\mathrm{~L}=\mathrm{g} \cdot \mathrm{T}^{2} / 2 \pi \cdot \operatorname{tg} \mathrm{h}(2 \pi \mathrm{d} / \mathrm{L})$ & $\mathrm{v} \cong \mathrm{cte}$ \\
\hline
\end{tabular}

Considerando la velocidad horizontal del agua en función de la profundidad relativa, se tiene:

\begin{tabular}{|c|c|}
\hline Para $d / L>1 / 25$ & Para d $/ L<\mathbf{1} / \mathbf{2 5}$ \\
\hline$v \cong 0$ & $v \cong$ cte \\
$\begin{array}{l}\text { La velocidad del agua es prácticamente nula. } \\
\text { No se realiza ningún cálculo. }\end{array}$ & $\begin{array}{l}\text { La velocidad es constante para toda la } \\
\text { profundidad. }\end{array}$ \\
\hline
\end{tabular}

De la fórmula para la zona de transición $\mathrm{L}=\mathrm{g} \cdot \mathrm{T}^{2} / 2 \pi \cdot \operatorname{tg} \mathrm{h}(2 \pi \mathrm{d} / \mathrm{L})$, obtenemos los datos $\mathrm{T}, \mathrm{L}, \mathrm{d}$.

Con las características del oleaje se calcula la estabilidad de la estructura, utilizando la propuesta de Djounkovski y Bojitch.

$$
G(f \cos \alpha-\operatorname{sen} \alpha)=S \varpi \pi h 2 / L e^{-2 m / L}
$$

Donde:

$\mathrm{G} \cong$ peso del arrecife artificial por unidad de superficie

$\mathrm{f} \cong$ coeficiente de rozamiento entre arrecife y fondo $\cong 0,79 ; \alpha \cong$ pendiente del fondo en grados 
$\mathrm{V}=$ velocidad media de arrastre $=2 \mathrm{~m} / \mathrm{sg}$.

$\mathrm{Sv}=$ sup. media total de los paños de red $=350 \mathrm{~m}^{2}$

$\alpha=$ ángulo de ataque del paño $=30^{\circ}$

$\mathrm{Rc}=$ fuerza de rozamiento de los cables

$\mathrm{Rc}=\mathrm{k} \cdot \gamma \cdot \mathrm{D} \cdot \mathrm{L} \cdot \mathrm{V}^{\wedge} 2$

$\mathrm{k}=$ coef. de arrastre 0,8

$\gamma=$ densidad agua marina

$\mathrm{D}=$ diámetro de los cables $=0,02 \mathrm{~m}$.

$\mathrm{L}=$ long. del cable $80<\mathrm{L}<100 \mathrm{~m}$.

$\mathrm{Rp}=$ fuerza de rozamiento de las puertas $=0,25 \cdot \mathrm{Rr}$

$\mathrm{Ra}=$ fuerza de rozamiento del arrastre de la estructura, $\mathrm{Ra}=\mathrm{f} \cdot \mathrm{N}$

$\mathrm{f}=$ coef. rozamiento hormigón-arena $=0,79$

$\mathrm{N}=$ peso sumergido del módulo.

Para que se cumpla la condición de arrastre

$$
\mathrm{Pd}>\mathrm{R} \cdot \mathrm{V} / 75
$$

\section{Descripción de las obras}

\subsection{Proceso constructivo}

El proceso de construcción de este tipo de obras consta de tres fases, bien diferenciadas:

\section{- Construcción de los elementos \\ . Transporte por tierra y mar. \\ . Instalación y fondeo.}

Para la construcción de las estructuras existen dos alternativas: la construcción prefabricada o la ejecución directa en el puerto.

En la construcción prefabricada hay que tener en cuenta la localización de la fábrica: lo más cercana posible al puerto de embarque; además, es conveniente realizar una valoración económica comparativa del transporte de los elementos prefabricados a puerto frente a la construcción en éste. Si se opta por la construcción de las estructuras directamente en una zona portuaria, será conveniente estudiar algunos aspectos como:

- La superficie total disponible en la zona será lo suficientemente amplia para permitir todos los procesos de elaboración y montaje de las estructuras.

- La distribución del área portuaria en zonas de acopio de materiales, construcción de elementos, ensamblaje y montaje, elementos totalmente terminados, etc.
- Hay que tener en cuenta que los elementos, después de fabricados, deberán permanecer acopiados, al menos, 28 días.

- Prever y estudiar la vialidad del área portuaria, dejando calles, rotondas, etc., entre las distintas zonas de trabajo, de modo que permitan las operaciones de carga y maniobras de la maquinaria.

- Los muelles de embarque se elegirán en función de las características del buque y la carga a transportar.

- Estudiar los medios auxiliares disponibles en el puerto, así como los sistemas más apropiados de carga para cada tipo de estructura.

- La situación del puerto respecto de los puntos de fondeo se estudiará para minimizar los tiempos de transporte marítimo.

- Estudiar las facilidades que ofrezca el puerto en cuestiones administrativas como tarifas, trámites, permisos, etc.

\subsection{Transporte marítimo}

La elección del medio de transporte marítimo está en función de la envergadura del proyecto, esto es, del número de estructuras proyectadas.

Los sistemas tradicionales se pueden resumir en los siguientes :

1.- Buques cargueros de pequeño tonelaje, aproximadamente entre 1.500 - 5.000 t.p.m., que equivale a la suma de los pesos del combustible y carga .

Las características exigidas o más apropiadas para este tipo de obras son:

Puntales: en general los barcos destinados a la carga están provistos de estos medios de izada, permitiendo las operaciones de carga y descarga en puertos y muelles en los que no existen grúas de muelle. Los puntales trabajan según la relación entre la capacidad de carga de los mismos y el peso de la unidad de carga, con lo que serán exigibles puntales entre 10 - $15 \mathrm{t}$ para la correcta ejecución de los procesos de carga. Actualmente, gran cantidad de buques poseen medios de carga de a bordo, es decir, grúas de barco (pórtico, telescópicas, en ménsula, etc.).

Bodegas de carga corridas: o sea, no compartimentadas, que aumentan la facilidad operativa, permitiendo una mejor disposición de los módulos arrecifales, así como la barrida en planta y elevación-descenso desde las grúas o puntales.

Pequeño calado: facilita la maniobrabilidad en el fondeo 
$\mathrm{S} \cong$ coeficiente de seguridad; $\omega \cong$ peso específico del agua marina $\cong 1.035 \mathrm{~kg} / \mathrm{m}^{3}$

$\mathrm{h} \cong$ semialtura de la ola de cálculo

$\mathrm{L} \cong$ semilongitud de la ola de cálculo

$\mathrm{z} \cong$ profundidad

De aquí, se obtiene el coeficiente de seguridad (S).

\subsection{Acción dinámica de las corrientes submarinas fren-} te al vuelco, deslizamiento de la estructura

Una corriente submarina de velocidad $\mathrm{V}$ puede provocar contra la estructura sumergida una fuerza (Figura 2) de:

$$
\mathrm{F}=\mathrm{C}_{0} \mathrm{~A} \mathrm{~W}_{0} \mathrm{~V} 2 / 2 \mathrm{~g}
$$

siendo :

$\mathrm{C}_{0}=$ coeficiente de forma del arrecife

$\mathrm{C}_{0}=1$ circular

$\mathrm{C}_{0}=2$ cuadrado

$\mathrm{C}_{0}=3$ trabado

$\mathrm{A}=$ sección perpendicular a la corriente

$\mathrm{W}_{0}=$ densidad del agua del mar

$\mathrm{V}=$ velocidad de la corriente

La suma de resistencias que se oponen al empuje será :

$$
\mathrm{R}=\mathrm{N} \cdot \operatorname{tg} \varphi
$$

de donde :

$\mathrm{N}=$ peso del módulo sumergido

$\varphi=$ coeficiente de rozamiento hormigón - arena $=30^{\circ}$

Condición de deslizamiento $\mathrm{F}>\mathrm{R}$

Condición de vuelco $\mathrm{F} \cdot \mathrm{d}>\mathrm{N} \cdot \mathrm{e}$
5.3. Cálculo de la estabilidad de las estructuras frente al tiro de embarcaciones

Para calcular la estabilidad de los módulos arrecifales ante el posible empuje que puedan ejercer las embarcaciones, se tendrán en cuenta los siguientes parámetros:

$\mathrm{F}=$ fuerza de tiro de las embarcaciones.

$\mathrm{R}$ = sumatorio de fuerzas de rozamiento.

La fuerza de tiro de las embarcaciones queda definida por:

$$
\mathrm{Pd}=\mathrm{Pn} \cdot \mathrm{Cu} \cdot \mathrm{Cp} \cdot \mathrm{Cm}
$$

siendo :

$\mathrm{Pd}=$ potencia de arrastre de cálculo.

$\mathrm{Pn}=$ potencia nominal del arrastrero. Se obtiene del censo de la flota susceptible de faenar en las aguas objeto del proyecto.

$\mathrm{Cu}=$ coef. de utilidad (rendim. del motor) $0,7<\mathrm{Cu}<0,9$.

$\mathrm{Cp}=$ coeficiente de propulsión $=0,2 \mathrm{si}>3.000$ r.p.m.

$\mathrm{Cm}=$ coef. del estado de la mar $=0,8$, mar ligeramente agitado.

Fuerza de rozamiento total (R)

$$
R=[(R r+R c+R p) \cdot 0,3]+R a
$$

siendo:

$\mathrm{Rr}=$ Fuerza de rozamiento de la red de arrastre

$$
\mathrm{Rr}=191 \cdot \mathrm{d} / 1+\mathrm{V}^{\wedge} 2 \cdot \mathrm{Sv} \cdot \operatorname{sen} \alpha
$$

$\mathrm{d}=$ diámetro de los hilos de la red $=0,6 \mathrm{~mm}$.

$1=$ lado medio de las mallas $=40 \mathrm{~mm}$.

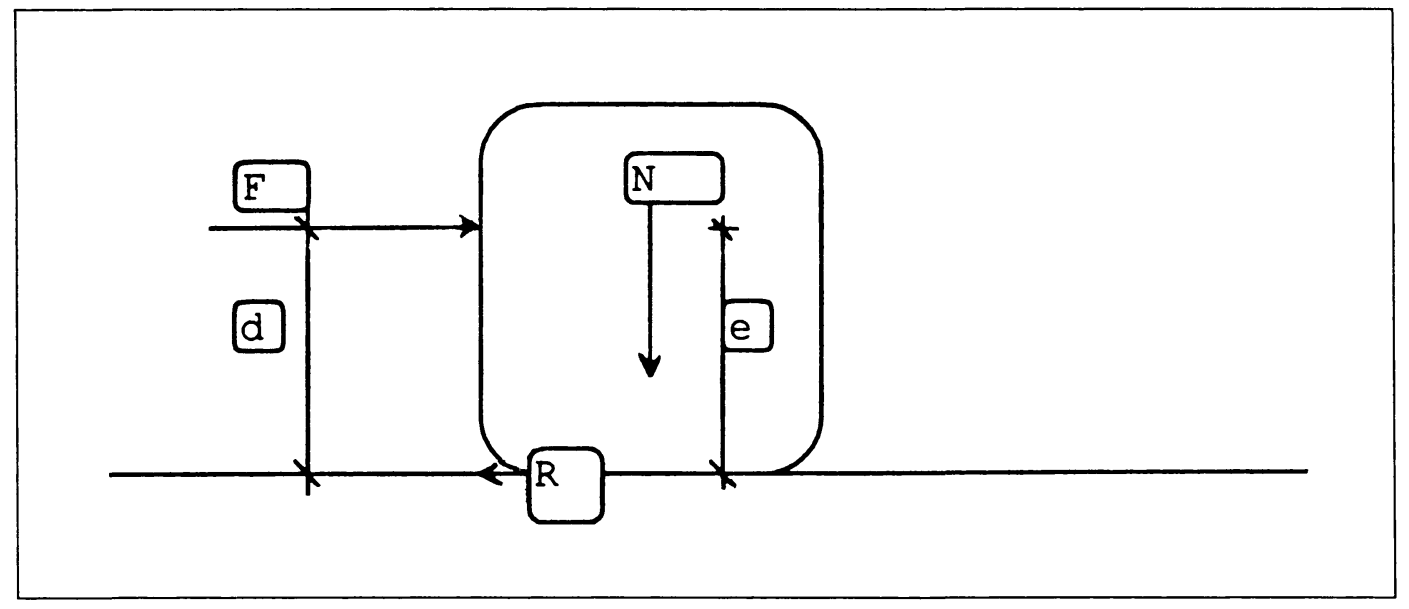

Figura 2. Diagrama de fuerzas que actúan sobre un elemento de un arrecife artificial. 
de las estructuras, así como los movimientos dentro del puerto, favoreciendo la entrada en puerto y economizando tarifas de atraque en muelles de menor calado.

Las dimensiones aconsejables de los buques para este tipo de obras serían:

eslora : $70-125 \mathrm{~m}$.

manga: $12-18 \mathrm{~m}$.

calado : $4-7 \mathrm{~m}$.

\subsection{Instalación y fondeo}

Antes de iniciar el fondeo es necesario realizar un replanteo, entendiendo éste como el conjunto de operaciones a realizar, encaminadas al correcto emplazamiento de los módulos en el fondeo. En el fondeo de los módulos se exigirá la máxima precisión posible en la instalación, por lo que se recomienda el uso de un sistema de posicionamiento global mediante satélites (GPS GLOBAL POSITIONINGSYSTEM).

Para corregir los errores producidos por fenómenos como: la inestabilidad de los relojes de los satélites, errores en las efemérides y en las órbitas, retrasos en la ionosfera y troposfera, multicaminos, ruidos, etc., se utilizará el Sistema Diferencial, consistente en el uso de dos equipos, uno en el barco y otro en tierra, ubicando, este último, en una base de coordenadas conocidas. Es conveniente el empleo de dos equipos de posicionamiento: uno, en el buque de carga y otro, en una embarcación auxiliar de apoyo. Con las comprobaciones precisas permite alcanzar precisiones de $2 \mathrm{~m}$. Tras el fondeo de cada módulo es conveniente realizar un balizamiento provisional de éstos, el cual permitirá tomar su posición real.

Una vez realizado el replanteo previo, se balizará el núcleo central, depositando las piezas a su alrededor. Para ello el barco deberá anclar en varios sitios, hasta coger la posición.

Se deberá tener precaución con el oleaje y las corrientes, los trabajos de montaje e instalación se suspenderán cuando la altura de ola sobrepase un límite (en general $\mathrm{H}=1 \mathrm{~m}$ ) tras el cual pueden existir riesgos de peligro ante el balanceo de las piezas, posibles golpes de las piezas con el buque e imposibilidad de guiado en el descenso. Las corrientes de fondo pueden desplazar los módulos, perdiendo el posicionamiento correcto, aun cuando en la superficie el buque se encuentre en la posición correcta.

El fondeo se realiza generalmente por dos sistemas diferentes:

- Caída libre desde la superficie.

- Descenso con cable y guiado por buceadores (Fotografía 5).

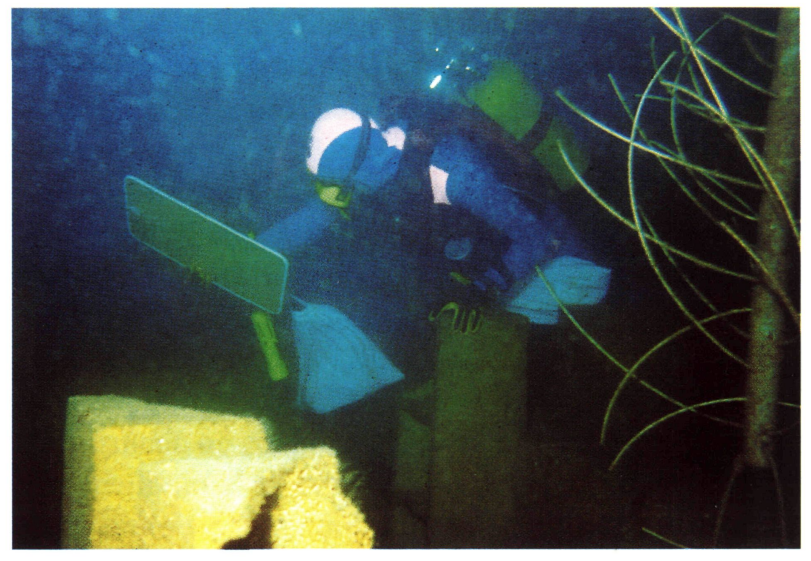

Foto 5.- Fondeo de estructuras mediante buceadores.

En el sistema de caída libre, recientemente utilizado, el buque, por medio de sus sistemas de carga eleva, el módulo y lo deposita en la superficie del mar, se suelta con un dispositivo de ganchos de disparo simultáneo, fondeándose el elemento por su propio peso. Si se opta por utilizar este sistema se deberán calcular las solicitaciones a que estará sometida la estructura al recibir el impacto sobre el fondo marino.

En el sistema de descenso con guiado por buceadores, se conjunta el fondeo con cable por medio de la ayuda de hombres rana. El buzo mediante un sistema de comunicación permanente con el barco, lo dirige, realiza los ajustes finales de las piezas y el desenganche definitivo. En caso de no ajustar o situar en un lugar incorrecto los módulos, debido a corrientes marinas o movimientos incontrolados del barco se utilizan globos de levantamiento que reducen el peso de las estructuras, permitiendo su colocación.

Entre los dos sistemas de fondeo existen una serie de ventajas e inconvenientes que inciden en la decisión final a adoptar para su instalación. El sistema de caída libre reduce riesgos generados por la permanencia de hombres en el fondo marino mientras se manejan las estructuras; el rendimiento en la colocación aumenta, reduciendo el tiempo de instalación; se reducen costes de conservación y mantenimiento de los sistemas de izado (cables, ganchos y demás dispositivos en contacto con el agua marina). Por otra parte, el sistema de descenso con ayuda de buceadores resulta imprescindible cuando se trata de estructuras que ensamblan entre sí o requieren un ajuste con mayor precisión. El estudio de los dos sistemas combinados parece ser la mejor solución, colocando los módulos de protección mediante caída libre y los módulos de producción con la ayuda de buzos.

\section{Seguimiento biológico}

La creación de arrecifes artificiales en el medio marino ha dado lugar a controversia por las posibles repercusiones ambientales sobre el medio marino. En este sentido, los 
estudios de Evaluación de Impacto Ambiental pueden servir para aclarar las dudas que existen. La construcción de arrecifes artificiales, ecológicamente, representa la aparición de nuevos ecotopos, con la consiguiente modificación de la composición y estructura de los ecosistemas presentes. Para Margalef (1992) la palabra impacto ambiental sugiere el carácter discontinuo, brusco y asimétrico que tiene, en general, la perturbación en relación con el cambio sucesional que se produce en un ecosistema. En el caso de arrecifes de reproducción y protección, parece evidente los cambios en cuanto a los ecotopos van a producir un aumento de la biodiversidad (aumento en la riqueza y proporción relativa de las diferentes especies de una biocenosis) (Fotografía 6); así como, cambios relativos a la estructura y función de los ecosistemas marinos, favoreciéndose la sucesión ecológica hacia ecosistemas más maduros, caracterizados por un aumento de la biomasa así como de las relaciones entre los distintos elementos de este sistema ecológico. Así pues, la presencia de estos arrecifes producen impactos positivos, continuos e irreversibles sobre los fondos

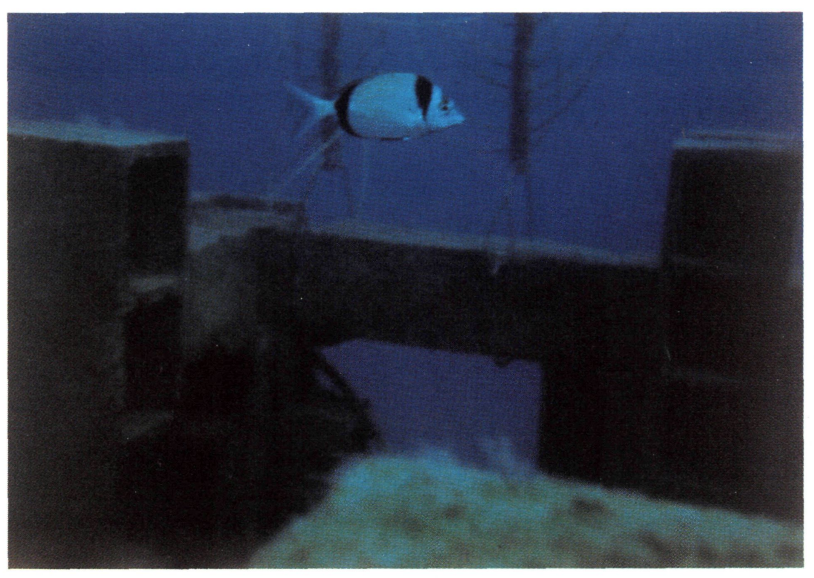

Foto 6.- Aparicion de nuevos animales en el arrecife.

marinos. Los planes de vigilancia ambiental sobre estas obras públicas, pueden ser, en el futuro, una solución que aclare esta cuestión al constituirse en nuevas fuentes de datos sobre la evolución de los fondos marinos.

\section{BIBLIOGRAFÍA}

- JENSEN, A. C. (Ed). 1996. European Artificial Reef Research. Proceedings of the $1^{\text {st }}$ earth conference. Ancona. Italia.

- LÓPEZ, A. y LÓPEZ, F. 1993. Aportes a la colonización, por algas bentónicas, del arrecife artificial de la isla de Tabarca (Alicante, España).Publicaciones Especiales del Instituto Español de Oceanografía.

- MARGALEF, R. 1992. Planeta azul, planeta verde. Ed. Omega. Barcelona.

- REVENGA, S., FERNÁNDEZ, F., GONZÁLEZ, J. L., SANTAELLA, E. 1996. Artificial reefs in Spain: the regulatory framework. En Jensen, A. C. (Ed). European Artificial Reef Research. Proceedings of the 1st earth conference. 1: 161-174. Ancona. Italy.

- SPGM: y Tecnologia Ambiental, S. A. 1995. Proyecto técnico de arrecifes artificiales para la protección de especies marinas de interés pesquero en cabo San Antonio (Alicante).

- SPGM: y Tecnologia Ambiental, S. A. 1995. Proyecto técnico de arrecifes artificiales para la protección de especies marinas de interés pesquero en cabo de Palos (Murcia). 